\title{
Développer l'approche par compétences dans la réforme 2013 du programme du DUT Communication des Organisations
}

Comment faire reconnaître l'intégration d'Internet dans ce DUT?

BOBIN Laurent, Consultant Webmarketing, fondateur de Becausse.com Agence Web en Aveyron, Chargé de cours à l'IUT de Rodez, laurent.bobin@becausse.com

VIDALENC Isabelle, Maître de conférences, Université Toulouse 1 Capitole, IUT de Rodez, Groupe MC2 (Médias, Communication et Culture) de I'IDETCOM, isabelle.vidalenc@iut-rodez.fr 


\title{
Résumé
}

En 2012, en France, un travail de réflexion sur les compétences attendues à l'issue du Diplôme universitaire de technologie (DUT) Communication des Organisations a été mené. Il a abouti à la création d'un référentiel de compétences. La logique des compétences s'impose ainsi comme une passerelle entre l'université et le monde du travail. A l'université, nous préparons les étudiants au développement d'une capacité réflexive et à leur inscription dans la logique de l'apprentissage tout au long de la vie. Or l'explicitation des compétences est loin d'être une opération évidente. P. Vermersch propose une méthode d'entretien d'explicitation. Appliquée aux savoirfaire du référentiel, elle permettra de déterminer les savoirs théoriques utiles pour compléter le programme pédagogique. En conclusion, le travail d'élaboration d'un programme pédagogique est un chantier jamais fini, où savoirs et savoir-faire interagissent pour toujours plus de dialogue entre les communautés universitaires et professionnelles. L'entretien d'explicitation sera alors utile pour faire émerger des compétences.

Mots-clefs : formation universitaire professionnalisante, DUT Communication des Organisations, référentiel de compétences, entretien d'explicitation, métiers de l'Internet

\begin{abstract}
In 2012, in France, a study on the competences awaited at the end of the technical degree (DUT) Communication of Organizations was led. It ended in a competency framework. It appeared that the logic of the competences stands out as a bridge between the university and the world of work. At the university, we prepare the students to develop a reflexive capacity and to enter into logic of learning throughout life. Yet the explicitation of the competences is far from being an obvious operation. P. Vermersch proposes a method of interview of explicitation which will be applied to the know-how of the competency framework, in order to identify the necessary theoretical knowledge to complete the educational program of the DUT. In conclusion, the work of an educational program is a never-ending process where knowledge and know-how interact for an ever greater dialogue between university and professional communities. The interview of explicitation will be a powerful tool to make competences come out.
\end{abstract}

Keywords: professional-qualification university education, communication of organizations, competency framework, interview of explicitation, Internet jobs 


\section{Le DUT et la réforme 2013 du programme}

Dans le cadre de l'harmonisation des cursus européens d'enseignement supérieur dite processus de Bologne, à partir de 1998, le système universitaire s'organise autour de trois diplômes ou grades : la Licence, le Master et le Doctorat (LMD ou 35-8). En France, il a été décidé de maintenir quelques diplômes nationaux aux côtés de ces nouveaux grades européens. C'est le cas du DUT, préparé en 2 ans, que les entreprises françaises ne souhaitaient pas voir disparaître car ce diplôme prépare les étudiants aussi bien à une insertion professionnelle à $\mathrm{Bac}+2$ dans les professions intermédiaires de technicien, qu'à la poursuite d'études courtes à $\mathrm{Bac}+3$ ou longues au niveau Master.

Construit à la fois par des universitaires et par des professionnels, depuis plus de quarante ans, le DUT offre aux étudiants des connaissances pluridisciplinaires solides et une culture professionnelle qui leur permet de répondre efficacement aux besoins des entreprises. Les IUT utilisent des méthodes de pédagogie active, études de cas, jeux de rôle, serious games, stages obligatoires, projets tuteurés. Organisés en réseau ${ }^{106}$, ils ont construit les plateformes Centrale-IUT ${ }^{107}$ et IUTenligne ${ }^{108}$ pour échanger sur ces pratiques pédagogiques innovantes.

En France, le DUT est un exemple de formation universitaire professionnalisante au niveau $\mathrm{Bac}+2$. Certaines expériences pédagogiques comme celle relatée dans l'article «Les dispositifs de professionnalisation des formations universitaires en communication » (Coyette et al, 2013) montrent l'intérêt d'une réflexion sur ces pratiques pédagogiques pour garantir l'équilibre entre les connaissances théoriques et les savoir-faire professionnels. Le dispositif pédagogique (appelé ci-dessous séminaire disciplinaire) mis en place par les auteurs a été comparé au référentiel de compétences établi par l'Association française des communicants internes (AFCI) en $2005^{109}$. Les auteurs (Coyette et al, 2013 : 105) tentent «par la mise en place de ce séminaire disciplinaire de répondre à la fois à la demande des professionnels du secteur, mais également de préserver l'apport de l'université (savoirs théoriques et capacités de réflexivité). » Cette démarche est à l'image de celle menée par les acteurs du DUT.

Une des spécificités du DUT est d'élaborer son contenu de formation suivant un Programme pédagogique national (PPN). Celui-ci est élaboré par les Commissions pédagogiques nationales (CPN) de spécialité, constituées de professionnels et d'enseignants, et les Assemblées de chef de départements (ACD). Elles sont les

\footnotetext{
$106 \quad$ http://www.iut.fr/ consulté le 28 septembre 2014

107 http://www.centrale-iut.net/ consulté le 28 septembre 2014

$108 \quad$ http://www.iutenligne.net/ consulté le 28 septembre 2014

109 http://www.afci.asso.fr/sites/default/files/pdf/publication/referentiel_afci.pdf consulté le 28 sept. 2014
} 
garantes de programmes en adaptation permanente à l'évolution des besoins du monde économique. Une concertation entre des enseignants et des professionnels impliqués dans la formation (chargé de cours, accueil de stagiaires, suivi de projets tuteurés) est organisée.

La réforme 2013 du PPN a permis d'adapter la formation aux conséquences induites par la réforme du baccalauréat en France et d'actualiser les compétences du DUT en les adaptant à l'évolution des technologies (la précédente réforme du PPN date de 2005). En décembre 2011, la Direction générale pour l'enseignement supérieur et l'insertion professionnelle (DGESIP) a demandé pour la première fois, en amont de l'écriture des contenus de formation, un travail de réflexion sur les compétences attendues à l'issue de la formation sous la forme d'un référentiel de compétences et activités.

Pour le DUT, la constitution de ce référentiel s'est basée sur les fiches du Répertoire opérationnel des métiers et emplois (ROME) de Pôle-Emploi ${ }^{110}$ correspondant aux spécialités, l'enquête nationale menée sur le devenir des diplômés de DUT $^{111}$ par la DGESIP et le réseau des IUT, ainsi que la consultation de professionnels du secteur.

En parallèle, le même travail a été mené pour le diplôme de licence générale selon la demande inscrite au nouvel arrêté du 1er août 2011. Ainsi, en juillet 2012, un référentiel de compétences définissant les objectifs de formation en termes d'acquis d'apprentissage suivant les champs disciplinaires des licences générales a vu le jour $^{112}$.

On peut s'interroger sur l'utilité de tels référentiels par rapport au décalage existant entre l'évolution constante des compétences identifiées dans chaque secteur professionnel et le délai de validation par les instances universitaires (dans notre cas, dix-huit mois). Notre objectif pour la construction du référentiel de compétences attendues après le DUT a été de clarifier le domaine professionnel couvert par la formation, aussi bien pour les futurs employeurs des diplômés que pour les futurs candidats, ainsi que leur famille. Nous avons créé une liste d'une dizaine d'activités, dans un ordre logique de réalisation des opérations, qui permette de se faire une idée assez complète et claire des tâches et missions attribuées au communicant des organisations (ci-après détaillées § 3.3). Ces activités ont été volontairement choisies parce qu'elles sont stables dans le temps, comme par exemple « Actions de relations

\footnotetext{
110 http://www.pole-emploi.fr/candidat/le-code-rome-et-les-fiches-metiers@/suarticle.jspz?id=15734 consulté le 21 septembre 2014

111 http://www.iut.fr/publications/le-devenir-des-etudiants.html consulté le 21 septembre

2014

112 http://www.enseignementsup-recherche.gouv.fr/cid61532/consultation-sur-les-

referentiels-licence.html consulté le 12 septembre 2014
} 
publiques ». Le référentiel de compétences ainsi créé doit avoir une existence valide d'environ six ans, délai dans lequel le programme pédagogique pourra être modifié.

Ce référentiel de compétences a donné un cadre pour établir le nouveau programme pédagogique. Il a montré son utilité pour permettre la création de nouveaux modules. Par exemple, le nouveau module «Culture numérique » est basé sur les compétences en «Informatique et culture numérique» identifiées dans le référentiel. Chaque module du nouveau programme a ainsi été situé par rapport aux activités du référentiel, comme par exemple, «Communication numérique » relève de l'activité «Conception d'une stratégie de communication » déclinée pour le web. Chaque enseignant pourra structurer ses interventions grâce aux objectifs pédagogiques identifiés dans le nouveau programme en termes de compétences (savoir et savoir-faire) à faire acquérir et grâce au référentiel de compétences et d'activité qui sert d'objectif final pour l'ensemble de la formation. Ce cadre un peu rigide ne l'empêchera pas de faire évoluer le contenu de ses interventions en fonction de l'évolution de la société et de la technologie. Il est même de son devoir de garder le cap donné par ce référentiel (figé pour six ans) tout en faisant évoluer chaque année le contenu des modules, si le besoin apparaît évident.

Enfin, dans le marché européen de la formation qui est en train de se constituer, ces référentiels donnent leur place et une meilleure visibilité à chaque diplôme. Cela doit permettre de conforter l'harmonisation de l'enseignement supérieur en Europe voulue par le processus de Bologne. De plus, le responsable d'un diplôme qui peut avoir à délivrer ce diplôme par Validation des acquis de l'expérience (VAE) ${ }^{113}$ pourra se baser sur ces référentiels de compétences pour clarifier les attendus universitaires auprès des candidats professionnels.

Avant d'analyser l'intégration des compétences liées aux métiers de l'Internet dans le nouveau programme pédagogique, le concept de compétences, d'un emploi récent dans le monde universitaire, mérite d'être développé. Nous définirons le concept de compétence, notamment ses rapports avec l'expérience et la connaissance. Nous mettrons en valeur la diffusion de la logique des compétences dans toute la société actuelle et le changement qu'elle apporte dans le monde universitaire. Ensuite, sur la base du constat que l'identification des compétences est loin d'être évidente, nous présenterons une méthode d'entretien qui pourra nous aider dans cette approche. 


\section{L'approche par compétences}

En France, nous assistons à une évolution importante des missions de l'université. Depuis la loi sur les libertés et les responsabilités des universités (dite LRU) du 10 août $2007^{114}$, la $3^{\mathrm{e}}$ mission de l'enseignement supérieur est, après la recherche et l'enseignement, l'orientation et l'insertion professionnelle. Tout est ainsi fait pour que la dynamique des compétences entre à l'Université et bouscule les missions traditionnelles de création et transmission du savoir. L'enseignant est notamment amené à reconsidérer les objectifs de ses cours en termes de compétences (savoir, savoir-faire et savoir-être) que doivent acquérir les étudiants. La logique des compétences s'impose ainsi comme une passerelle entre les études et le monde du travail.

\subsection{Définition de compétence}

L'Agence nationale pour l'amélioration des conditions de travail (ANACT) définit la compétence comme : «La combinaison de ressources mobilisées par l'individu dans une situation de travail ». Le Mouvement des entreprises de France (MEDEF) est plus précis dans la définition des ressources mobilisables : " La compétence est une combinaison de connaissances, savoir-faire et comportements, s'exerçant dans un contexte précis ». Ainsi la compétence est en lien avec les connaissances. Il convient donc de préciser le sens du mot connaissance.

Les philosophes ont longuement débattu sur la connaissance. Le concept est complexe. Étymologiquement, connaître est associé à comprendre par le fait qu'ils sont issus de la même racine latine nosco : être familiarisé avec, être informé. Par ailleurs, il est tentant de suivre Paul Claudel (Claudel, 1907 : 182) dans son jeu de mot avec «naître » : « Se connaître, c'est se fournir comme moyen de co-naissance, c'est pour l'être vivant faire naître en tant qu'avec soi tous les objets dont il a connaissance et dont il est l'image commune. » Nous connaissons les choses, nous les « co-naissons " dans une forme de renaissance permanente. Ainsi, nous choisissons de considérer la connaissance comme une transformation de la personne par la compréhension théorique et/ou pratique de quelque chose de nouveau.

Avec le concept de compétence, on ajoute à la personne la capacité à mobiliser ses connaissances (savoir, savoir-faire) et ses attitudes comportementales (savoir-être) en situation, dans un contexte précis. Par exemple, je connais le droit commercial (savoir acquis à l'université) mais cela ne me suffit pas pour traiter un dossier de contentieux (compétence acquise en stage). Je sais ce qu'est un dossier de

114 http://www.enseignementsup-recherche.gouv.fr/cid55933/presentation-autonomie-desuniversites.html consulté le 12 sept 2014 
contentieux mais tant que je n'en ai pas traité un précis en situation réelle, je n'en ai pas la compétence.

Les compétences peuvent être mesurées, selon ce que l'on est capable de faire en situation :

- Notion : je découvre, je m'initie, je suis capable de réaliser une activité avec de l'aide.

- Application : je pratique, je sais faire, je suis capable de réaliser une activité en autonomie.

- Maîtrise : je consolide, je maitrise, je suis capable de réaliser une activité en autonomie et de transmettre des savoirs et savoir-faire.

- Expertise : je fais évoluer, je forme, je suis capable de réaliser une activité en autonomie, de transmettre des savoirs et savoir-faire et de les faire évoluer.

Dans la continuité des propos tenus par R. Wittorski lors du Symposium «Écrire la compétence en information et communication» à Roubaix en Octobre 2009 (Wittorski, 2009: 4), nous distinguons le sens de compétence au singulier, qui est utilisé depuis longtemps, "être compétent» qui signifie être expert et être reconnu performant dans un domaine, du sens plus récent de compétence au pluriel : «avoir des compétences » qui signifie avoir l'aptitude, la capacité de mobiliser des savoirs et savoir-faire en action.

L'économie, les systèmes d'échange, de production et de commerce ont considérablement changé en peu de temps. L'entreprise est toujours plus contrainte à relever des défis, à s'adapter aux aléas du marché et de la concurrence, à anticiper les mutations techniques et réglementaires. Les technologies évoluent sans cesse, à un rythme rapide, de nouveaux schémas d'organisations en découlent modifiant la réalité et la notion même de métiers. Les métiers ont des durées de vie variables et s'exercent dans des situations très différentes, un même intitulé cache des conditions d'exercice très variées.

La notion de qualification reste indispensable mais ne suffit plus pour rendre compte d'une activité professionnelle ni pour prédire une chance d'insertion dans le monde du travail. A la périphérie du métier de base se développent désormais des activités exigeant des compétences supplémentaires qui sont généralement transverses à différents métiers et à plusieurs secteurs d'activités (Colin et Mouillet, 2000 : 9). Laissons R. Wittorski (Wittorski, 2009 : 4) conclure :

Compétences au pluriel intervient dans un contexte de crise (...) où il s'agit finalement de tenir le discours selon lequel les formes de travail évoluent rapidement parce qu'il y a plus de concurrence, parce qu'il s'agit de se démarquer des autres, parce qu'il s'agit de faire face à un marché qui évolue rapidement. (...) Je crois que l'apparition du mot compétences au pluriel accompagne une nouvelle conception du professionnel. En observant des discours sociaux tenus par des entreprises, il y a un peu l'idée que le professionnel d'aujourd'hui ce n'est plus le professionnel discipliné de l'ère 
taylorienne c'est un professionnel qui est capable d'opérer un changement là où il se situe (...) d'adapter ce qu'il fait et de communiquer à propos de son faire.

\subsection{Transférabilité des compétences : nouvelle conception du professionnel et de l'étudiant}

L'organisation de toute entreprise définit le travail des opérateurs par un ensemble de prescriptions officielles et normatives qui déterminent les objectifs et moyens assignés à l'opérateur pour fournir une production donnée : c'est ce que l'on appelle la tâche prescrite. Cependant, l'analyse du travail en situation réelle montre que l'opérateur n'exécute pas exactement cet ensemble de prescriptions. En effet, l'opérateur doit gérer et résoudre un ensemble de contraintes et problèmes non prévus par la tâche prescrite : c'est ce que l'on appelle la «tâche réelle » (Haulet et al, 2002).

Dans les nouveaux modes d'organisation, l'écart entre tâche prescrite et tâche réelle est de plus en plus large. Les objectifs sont précis mais les moyens à mettre en œuvre le sont peu. La responsabilité de s'organiser, de s'adapter à la tâche, de savoir agir est transférée vers l'individu, quelque soit son niveau hiérarchique. C'est dans cet écart que se nichent les compétences individuelles acquises pour la plupart hors cursus de formation. Ces compétences nécessaires sont devenues un thème central en matière de ressources humaines, elles sont repérées, évaluées, gérées, référencées, c'est là l'objectif de la Gestion prévisionnelle des emplois et des compétences ${ }^{115}$.

Dans un environnement professionnel devenu complexe, très évolutif et imprévisible, l'individu devra fréquemment faire des choix et changer de direction. Les opportunités de trouver un emploi, d'en changer, d'évoluer dans l'entreprise existent, elles sont même nombreuses, mais pas forcement visibles, d'où la nécessité pour chacun d'avoir explicité ses compétences personnelles et de savoir les réinvestir dans des contextes différents (Colin et Mouillet, 2000: 10). Cette approche par compétences est bien mise en évidence dans le ROME (Répertoire opérationnel des métiers et des emplois) qui, dès 1993, a substitué à une logique des emplois statutairement accessibles, une logique d'aire de mobilité professionnelle (Ruedin, 2001), définie sans égard pour le niveau de qualification mais en regard à la proximité entre les emplois définie par les compétences transférables, la similitude des compétences, pour une recherche d'adaptabilité. C'est aussi ce que nous dit A. Kiyindou lors du Symposium «Écrire la compétence en information et communication » à Roubaix en Octobre 2009 (Kiyindou, 2009 : 52) :

115 http://www.anact.fr/web/dossiers/travail-developpement-des-personnes/gpec consulté le 24 septembre 2014 


\begin{abstract}
L'idée de compétences accompagne donc une nouvelle conception du professionnel perçue avant tout comme un praticien réflexif puisque la compétence est liée à l'action, l'activité du sujet doit viser l'adaptation mais l'action avec sa part motivationnelle doit être assumée par l'individu. On peut également voir derrière ce dispositif une volonté sinon un risque de responsabilisation de l'individu par rapport à sa réussite professionnelle. Au delà de la mise en ordre, la question de la compétence est une question de mesure mais aussi de mise en concurrence. Elle ne s'arrête pas avec l'Université, mais s'inscrit dans une logique d'apprentissage tout au long de la vie.
\end{abstract}

A l'université, nous avons le devoir de préparer les étudiants au développement de cette capacité réflexive, à l'explicitation de leurs compétences et à leur inscription dans cette logique de l'apprentissage tout au long de la vie, qui est garante de leur capacité à s'adapter en permanence aux nouvelles technologies et aux changements du monde économique. Tout ceci est prévu dans les dispositifs de développement du projet professionnel et personnel de l'étudiant, inclus depuis 2005 en DUT et 2007 dans toutes les formations de niveau licence. Dans tous les diplômes universitaires français jusqu'au grade de Licence, dans le but d'améliorer leur réussite, les étudiants ont à faire un bilan personnel de leurs atouts et faiblesses, et à envisager leur avenir professionnel (métier et poursuite d'études). Les étudiants vont être amenés à découvrir le monde professionnel, les vraies réalités des métiers, par le biais d'interviews et de recherches d'information. Ils vont aussi chercher à mieux se connaître, entrer dans une réflexion personnelle nourrie de l'analyse de leurs propres expériences et ainsi prendre conscience de leurs compétences et aptitudes, et de leur besoin de progresser.

L'étudiant va être guidé dans la réalisation de son portefeuille de compétences, c'est-à-dire identifier, décrire et analyser ses compétences acquises lors de formations, d'expériences sociales et professionnelles (emploi, stage, loisir, bénévolat, travail familial, etc.). Pour obtenir un outil efficace et opérationnel, il est nécessaire d'extraire les savoirs, savoir-faire et savoir-être de chaque expérience, quelle qu'elle soit. Or l'identification des compétences est loin d'être une opération évidente, ni pour les étudiants, ni pour les professionnels.

En effet, l'action est une connaissance autonome. Le sujet pour réussir son action n'a pas besoin de savoir qu'il sait. C'est-à-dire qu'il n'a pas besoin d'avoir conscience des moyens de sa réussite pour réussir. Cela se traduit par la réponse « Je ne sais pas » à la question «Comment faites-vous?». On peut dire que l'action est opaque à celui-là même qui l'accomplit.

Dès 2005, à l'IUT de Rodez, il a été décidé d'utiliser l'entretien d'explicitation (Vermersch, 2011) dans les départements Information-Communication et Informatique pour aider les étudiants à prendre conscience de l'ensemble des compétences apportées par des expériences apparemment banales. Ce dispositif pédagogique a été présenté dans l'article "L'entretien d'explicitation : Un outil performant pour l'analyse des compétences » (Malric et Vidalenc, 2013). 


\subsection{Un outil pour l'explicitation des compétences}

L'entretien d'explicitation est une technique d'aide à la verbalisation a posteriori d'une activité/tâche réalisée par une personne (appelée ci-après le sujet), tant au niveau des actions matérielles que mentales. S'il s'appelle «entretien d'explicitation ", c'est pour bien indiquer que cette technique d'entretien permet au sujet d'expliciter ce qui est non conscient dans le déroulement de son activité. P. Vermersch a développé une véritable technique d'entretien originale qui aide à l'évocation du passé, à l'émergence de l'implicite, à la verbalisation et à la prise de conscience des compétences, qui écarte les jugements pour se concentrer sur les faits. A la fin de l'entretien d'explicitation, l'interviewer et l'interviewé doivent avoir acquis des informations sur la manière dont ce dernier a réalisé une tâche particulière.

L'entretien d'explicitation permet la décomposition et la prise de conscience de compétences qui pourront être réinvesties dans diverses situations d'emploi, de stage ou de vie sociale. Les compétences sont d'autant plus transférables que le niveau de décomposition est fin. L'interviewer doit oublier tout ce qu'il croit savoir pour obtenir par ses questions la verbalisation de toutes les actions par l'interviewé.

Voici deux exemples de compétences transférables découvertes grâce aux entretiens d'explicitation : une grande habileté à manipuler le joystick renvoie aux jeux vidéo mais est essentielle pour un conducteur d'engin de chantier, un manipulateur de matière radioactive ou de virus et aussi pour le chirurgien qui opère sans ouvrir. Ou encore, le fait d'avoir pratiqué un sport d'équipe en extérieur ou d'avoir fait les vendanges démontre une compétence transférable dans le travail sous les intempéries, monteurs de lignes, maraîchers, géomètres, ingénieurs des travaux publics ou conducteurs de travaux.

Et ci-dessous, un exemple réel d'expérience d'étudiant en DUT $1^{\text {ière }}$ année Informatique, développée tout d'abord spontanément en savoir, savoir-faire et savoir-être, puis décomposée grâce à l'entretien d'explicitation.

Alexandre : «Cet été j'ai travaillé en tant que manutentionnaire. J'ai rangé une salle d'archives complète avec une autre personne de mon âge. Nous avons monté environ 500 mètres linéaires d'étagères. J'ai eu un CDD de 1 mois qui a été prolongé pour que je monte une $2^{\mathrm{e}}$ salle. J'ai également assemblé quelques livres ». 
166 Le communicateur bousculé par le numérique

\begin{tabular}{|l|l|l|}
\hline SAVOIR & SAVOIR FAIRE & SAVOIR ETRE \\
\hline Manutention & Manutention & Mieux travailler en équipe \\
\hline
\end{tabular}

TABLeAU 3

Développement spontané

\begin{tabular}{|c|c|c|}
\hline SAVOIR & SAVOIR FAIRE & $\begin{array}{l}\text { SAVOIR } \\
\text { ETRE }\end{array}$ \\
\hline $\begin{array}{l}\text { Le système de montage du } \\
\text { rayonnage }\end{array}$ & $\begin{array}{l}\text { Installer les montants } \\
\text { Fixer les tablettes aux } \\
\text { montants }\end{array}$ & Méthodique \\
\hline $\begin{array}{l}\text { Les différentes catégories } \\
\text { de produits à archiver } \\
\text { Le système de } \\
\text { référencement } \\
\text { Le système de classement }\end{array}$ & $\begin{array}{l}\text { Positionner les archives } \\
\text { sur le rayonnage } \\
\text { Respecter le classement }\end{array}$ & $\begin{array}{l}\text { Patient } \\
\text { Endurant } \\
\text { Habile et agile }\end{array}$ \\
\hline $\begin{array}{l}\text { Les règles de posture pour } \\
\text { la manutention }\end{array}$ & $\begin{array}{l}\text { Évaluer la quantité à } \\
\text { déplacer en une fois en toute } \\
\text { sécurité } \\
\text { Placer l'échelle de manière } \\
\text { optimale }\end{array}$ & Ponctuel \\
\hline $\begin{array}{l}\text { Les règles de sécurité pour } \\
\text { les locaux } \\
\text { Les méthodes d'assemblage } \\
\text { des livres }\end{array}$ & Organiser la collaboration & $\begin{array}{l}\text { Organisé } \\
\text { Collaboratif }\end{array}$ \\
\hline
\end{tabular}

\section{TABLEAU 4}

Décomposition qui peut-être obtenue à minima par l'entretien d'explicitation

P. Vermersch (Vermersch, 2011, p .45) éclate le concept d'action en cinq facettes selon un axe vertical et un axe horizontal. La catégorie du procédural est le cœur du 
système et les quatre autres catégories en sont les satellites. Voici le schéma qu'il propose pour analyser l'action :

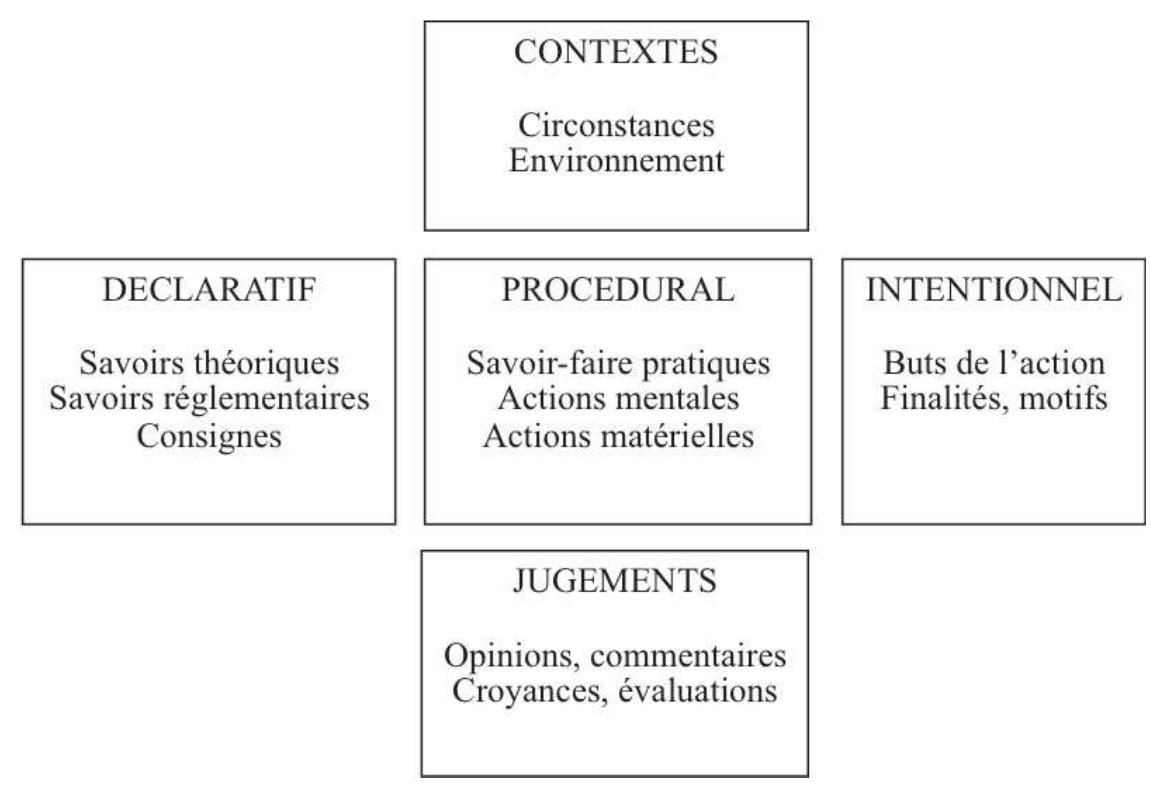

\section{FIGURE 16}

\section{Eclatement de l'action en cinq facette selon P. Vermersch (2011, p. 45)}

Sur l'axe horizontal du schéma ci-dessus, sont abordés les rapports entre les savoirs théoriques, les buts de l'action et l'action elle-même. Ce qui est original dans l'entretien d'explicitation, c'est de partir de la dimension procédurale pour inférer les savoirs théoriques effectivement mis en œuvre, pour découvrir quels sont les savoirs qui sont réellement utilisés dans l'action. Lorsque l'on interroge le sujet directement sur ses connaissances théoriques, rien ne permet d'être certain que ce sont des connaissances qu'il utilise réellement dans l'action.

Cette méthode va nous permettre, à partir des savoir-faire identifiés dans le référentiel de compétences et d'activités, d'identifier les savoirs théoriques nécessaires et ainsi compléter le programme de formation du DUT. Pour mettre en valeur l'intégration d'Internet dans ce nouveau programme, nous allons plus particulièrement analyser les compétences liées aux métiers de l'Internet. Nous nous appuierons sur l'ancien programme, le portail des métiers de l'Internet, le nouveau 
168 Le communicateur bousculé par le numérique

programme et l'expérience de deux nouveaux cours menés à l'IUT de Rodez en 2014.

\section{L'intégration d'Internet dans le nouveau programme pédagogique}

\subsection{Bilan de l'ancien programme}

Depuis 2005, date du précédent PPN, la spécialité Communication des organisations (Com) du DUT ne rencontre pas de problèmes de recrutement ni de placement des étudiants en stage. Ce DUT fonctionne bien, les étudiants, s'ils le souhaitent, peuvent s'insérer dans le monde du travail ou bien continuer leurs études. La seule modification à lui apporter sera d'intégrer Internet, les Technologies de l'information et de la communication (TIC), les médias numériques et les compétences liées à la communication sur le web de façon visible. En effet, seul le module de formation «Multimédia PAO» de l'ancien PPN permettait de former aux fonctions de webmaster éditorial. Par la suite, en 2009, certains modules complémentaires étaient plus nettement orientés Internet: «Écritures professionnelles », « Nouvelles techniques de rédaction», «Publication web», «Écritures numériques» et «Ateliers de réalisation Internet». Mais ces modules complémentaires étant optionnels, tous les étudiants n’y avaient pas accès.

Voici, ci-dessous, le tableau de savoirs et savoir-faire que nous avons créé à partir du PPN 2005, complété en 2009. Suivant les principes de P. Vermersch, un travail d'explicitation des savoirs et savoir-faire a été mené à partir des courts descriptifs des modules fournis. On peut remarquer qu'un certain nombre de compétences liées à Internet avaient alors bien été identifiées. Il convient, en 2013, d'afficher plus clairement que tous les étudiants du DUT Com seront formés à la communication web. 


\begin{tabular}{|c|c|}
\hline $\begin{array}{l}\text { Savoir-faire du PPN 2005-2009 } \\
\text { associés à Internet }\end{array}$ & $\begin{array}{l}\text { Savoirs du PPN 2005-2009 } \\
\text { associés à Internet }\end{array}$ \\
\hline $\begin{array}{l}\text { Utiliser les techniques de base de } \\
\text { l'écriture multimédia } \\
\text { Adapter l'expression écrite au support } \\
\text { web } \\
\text { Capter l'attention des lecteurs du web } \\
\text { Adapter les stratégies éditoriales en } \\
\text { fonction du support }\end{array}$ & $\begin{array}{l}\text { Stratégies rédactionnelles } \\
\text { Principes de conception d'un message } \\
\text { multimédia } \\
\text { Titre, titraille } \\
\text { Genres journalistiques } \\
\text { Comportement des lecteurs sur le web } \\
\text { Principes d'adaptation d'un message } \\
\text { aux différents publics }\end{array}$ \\
\hline $\begin{array}{l}\text { Réaliser un cahier des charges d'un } \\
\text { produit d'information sur Internet } \\
\text { Concevoir un produit d'information } \\
\text { sur Internet }\end{array}$ & $\begin{array}{l}\text { Principes de gestion d'un projet de } \\
\text { production d'un produit d'information } \\
\text { sur Internet }\end{array}$ \\
\hline $\begin{array}{l}\text { Évaluer les outils de publication du } \\
\text { web } \\
\text { Évaluer les outils de communication } \\
\text { numérique }\end{array}$ & $\begin{array}{l}\text { Outils de publication du web } \\
\text { Outils de communication numérique } \\
\text { du web } 2.0\end{array}$ \\
\hline $\begin{array}{l}\text { Administrer un gestionnaire de } \\
\text { contenu (CMS) } \\
\text { Créer des pages html à l'aide d'un } \\
\text { outil d'édition web } \\
\text { Mettre en œuvre et utiliser un outil } \\
\text { interactif du Web 2.0 (wiki, CMS, blog) }\end{array}$ & $\begin{array}{l}\text { Principes du CMS } \\
\text { Principes du langage html }\end{array}$ \\
\hline
\end{tabular}

\section{TABLEAU 5}

Tableau des savoirs et savoir-faire créé à partir du PPN

\subsection{Analyse des métiers de l'Internet}

Le secteur du web est un secteur d'activité innovant, en pleine croissance et porteur d'emplois : des métiers se créent au fur et à mesure que se développent les technologies et les nouveaux services et usages du réseau. C'est pour favoriser une meilleure connaissance de ces métiers et répondre aux besoins de compétences des acteurs de l'économie numérique que la Délégation aux usages de l'Internet a créé un portail des métiers de l'Internet ${ }^{116}$. Sur ce site, l'Office national d'information sur 
les enseignements et les professions (ONISEP), partenaire de la Délégation, a identifié pour le DUT Com les deux métiers de l'Internet suivants :

- Chargé de communication web : stratégie de communication interne et externe sur le web, élaboration des contenus, mise en œuvre du plan d'actions de communication sur le web ;

- Chargé des relations publiques digitales: entretenir les relations entre l'organisation et ses publics par le biais d'Internet, influence, e-réputation, garant de la cohésion de l'ensemble des actions de communication sur le web.

Tous deux apparaissent clairement comme des transpositions sur Internet des métiers traditionnels de Chargé de communication et Chargé de relations publiques. Les compétences traditionnelles, notamment tout ce qui relève de la stratégie de communication, de l'élaboration des contenus, de la cohésion des actions, sont déclinées, mais pas révolutionnées, sur un nouveau support : le numérique, aussi libellé Internet ou web ou digital.

Par ailleurs, un nouveau métier apparaît comme emblématique de l'essor du numérique au sein de la fonction communication, celui de community manager ou animateur de médias sociaux. Dans leur article «Community management et community managers: Cheval de Troie marketing pour le web social?», A. Coutant et T. Stenger (Coutant et Stenger, 2011) montrent que «le community manager est apparu comme le nouveau métier chargé de relever tous les défis liés à la présence des marques/entreprises au sein des médias sociaux ». Ils nous rappellent que l'origine de ce métier est liée à l'univers de l'administration des jeux vidéo en ligne et des forums, avec la fonction de modérateur des commentaires, et surtout à l'esprit communautaire d'Internet. Puis viennent les blogs avec la figure emblématique du «bloggeur influent» et la logique de «viralité » qui lui est associée, puis la période actuelle des médias sociaux avec le leader FaceBook, le micro-blogging Twitter et les sites de networking (réseautage professionnel) LinkedIn et Viadéo.

Le community manager développe la présence de l'organisation (marque, association, personnalité...) dont il se fait le porte-parole sur les médias sociaux, il est le médiateur entre les internautes membres de la communauté et les dirigeants de l'organisation, et le relais des contributions, critiques, avis, idées et suggestions des membres. C'est donc bien un communicant pour l'organisation qui le rémunère.

Cette analyse nous permet de bien identifier le profil particulier de ce nouveau métier qui est inscrit dans le PPN 2013 mais qui n'est pas présenté comme un débouché du DUT Com sur le portail des métiers de l'Internet. On peut se demander pourquoi, d'autant plus que son responsable hiérarchique, le Gestionnaire des médias sociaux, est présenté comme un véritable «responsable des relations publiques de l'organisation sur le web »... Nous retrouvons donc le chargé des relations publiques digitales et nous avons en plus la preuve de la difficulté 
d'harmonisation des libellés de métiers et de niveau de description des compétences. Nous pouvons regretter que ces deux libellés existent pour les mêmes compétences.

Ainsi, nous voyons que le DUT Com peut conduire aux métiers liés aux médias sociaux, considérés comme un nouveau média, un nouveau support de communication et d'information nécessaire notamment à la fonction de chargé de relations publiques. Sur le portail des métiers de l'Internet, ces quatre métiers (qui ne sont que trois au final) font partie de la même famille : Communication et Marketing.

Les étudiants du DUT Com peuvent aussi prétendre à la famille Interfaces et création numérique, avec les métiers Webdesigner et Graphiste web. Et enfin dans la famille: Production et gestion de contenu, ils peuvent prétendre au métier de Rédacteur web, Responsable éditorial online et Webmestre, le responsable du site Internet ou Intranet, qui peut travailler aussi bien sur l'administration du site que sur la ligne éditoriale ou sur le graphisme. Ces métiers sont identifiés dans le PPN 2013 mais ne sont pas reconnus par l'ONISEP en tant que débouchés du DUT Com.

Pour clarifier les nouveaux débouchés du DUT Communication des organisations, les nouveaux métiers tels que concepteur de site web (fiche ROME E1104 Conception de contenus multimédias) et animateur de médias sociaux (fiche ROME E1101 Animation de site multimédia) ont été ajoutés à ceux plus traditionnels de chargé de communication, chargé de « relations publics » ${ }^{117}$, chargé de relations presse, webmaster (fiche ROME E1103 Communication) ou chargé de communication événementielle/chef de projet événementiel (fiche ROME E1107 Organisation d'événementiel). Toutes les descriptions de ces métiers ont été utilisées pour en extraire les savoir-faire, savoirs et savoir-être du référentiel de compétences et activités que nous allons maintenant présenter.

\subsection{Référentiel de compétences et les nouveaux cours du PPN 2013}

Dans ce référentiel, nous avons exprimé par des verbes d'action des savoir-faire auxquels le nouveau DUT Communication des organisations devra préparer les étudiants. Les savoirs n'ont pas été intégrés au référentiel de compétences, ils seront identifiés dans un second temps, lors de la constitution du référentiel de formation, suivant en cela l'idée que les savoirs sont ceux utilisés pendant l'activité professionnelle, comme le préconise P. Vermersh, et aussi ceux identifiés par les

$117 \quad$ Notons le changement de nom, décidé lors de l'assemblée générale extraordinaire du syndicat de la profession, Syntec Conseil en relations publics, le 7 juin 2011, pour clarifier la fonction de relation avec les publics et la distinguer des relations en public et de la communication publique. 
enseignants pour permettre aux étudiants de poursuivre leurs études universitaires, comme par exemple le cours de Théories en sciences de l'information et de la communication.

Les savoir-être ont été extraits des fiches-métiers et exprimés sous une forme rédigée: Le diplômé doit faire preuve d'une grande aisance relationnelle et comportementale, être curieux du monde qui l'entoure, avoir une sensibilité esthétique et artistique. Il est rigoureux, il détient un certain sens critique, il a le sens de l'organisation, il sait être à l'écoute des autres et il a le goût pour le travail en équipe.

Une liste d'une dizaine d'activités a été pensée dans un ordre logique de réalisation des opérations. Elle permet de se faire une idée assez complète et claire des tâches et missions attribuées au communicant des organisations ${ }^{118}$.

1. Construire un réseau professionnel ;

2. Concevoir une stratégie de communication (cibles, objectifs, supports et actions) ;

3. Mettre en œuvre une politique de communication ;

4. Concevoir et analyser de messages écrits, visuels, audios, audiovisuels, multimédia, web ;

5. Réaliser des produits et supports de communication écrits, visuels, audios, audiovisuels, multimédia, web ;

6. Gérer le côté matériel et logistique de la communication ;

7. Gérer des dispositifs de communication interne ;

8. Gérer des actions de relations «publics », médias, hors média et avec les TIC ;

9. Gérer des actions de relations presse ;

10. Gérer des actions de marketing à destination des clients et des actionnaires.

A cette liste il faut ajouter les activités communes aux cinq options du DUT Information-Communication :

11. Maîtriser l'expression écrite et orale en français ;

12. Maîtriser l'expression écrite et orale dans deux langues vivantes étrangères ;

13. Utiliser professionnellement l'informatique;

14. Avoir une culture du numérique et des TIC ;

15. Travailler en mode projet;

16. Analyser le monde contemporain grâce aux Sciences Humaines et Sociales.

On peut remarquer que l'activité de conception de messages et l'activité de réalisation de produits et supports de communication sont déclinées sous différentes formes : écrit, visuel, audio, audiovisuel, multimédia et web. On voit là apparaître toute la problématique de l'adaptation des messages au support et au public, qui

118 Pour une présentation détaillée de ces activités, il faut se reporter au PPN, pages 4-6 http://cache.media.enseignementsup-recherche.gouv.fr/file/30/15/0/Infocom_262150.pdf. 
n'est certes pas nouvelle mais simplement renouvelée avec le média Internet, symbolisée par le nouveau cours «Écrire pour le web ». « Outils de communication numérique » complète ce nouveau cours en formant les étudiants à la création d'un système de gestion de contenu (CMS) pour par exemple leur permettre de créer un journal en ligne, une newsletter, un blog, un forum.

Ensuite, l'activité de relations avec les publics évolue nettement avec Internet : en plus des techniques habituelles de communication média et hors média, le responsable des «relations publics» doit animer et manager une communauté, alimenter les blogs et médias sociaux, travailler leur référencement. Cette nouvelle conception des relations avec les publics donnera lieu à deux nouveaux cours «Webmarketing» et «Communication numérique», pour couvrir aussi bien l'aspect technique que l'aspect stratégie web.

Enfin, l'activité « Informatique et culture numérique » est apparue pour marquer la prise en compte de l'évolution de la société avec les nouveaux outils numériques d'information et de communication. Deux nouveaux cours ont été pensés «Outils informatiques » et «Culture numérique » pour faire connaître et comprendre aux étudiants les possibilités offertes par le média Internet et les TIC. Un nouveau cours « Multimédia et web » permet de montrer aux étudiants comment créer un site web avec intégration d'animation et de feuilles de style (CSS), et aussi comment mener un projet de création d'un site web avec rédaction du cahier des charges. Ce cours peut permettre d'aborder les bases de données et les sites web dynamiques (PHP).

\subsection{Un retour d'expérience sur les nouveaux cours « Culture numérique » et "Webmarketing »}

Nous allons maintenant faire le point sur l'expérience des nouveaux cours « Culture numérique » et « Webmarketing » mis en place en janvier 2014 à l'IUT de Rodez. L. Bobin, consultant webmarketing, nous fait part du positionnement des étudiants du DUT Communication des organisations concernant les trois composantes des métiers de la communication web, à savoir : Développement / Graphisme et Contenu / Marketing. Les affirmations qui suivent sont donc basées sur une analyse des réactions d'un groupe d'étudiants et du professionnel enseignant à l'application du nouveau programme du DUT. Elles mettent en valeur les compétences liées aux métiers de l'Internet.

Le développement web est le domaine le plus abscons pour les étudiants, celui vers lequel ils inclinent le moins. A raison, puisque leur choix de cursus est déconnecté de notions techniques informatiques et que le développement web n'est pas vraiment au programme. Les étudiants doivent prendre conscience que les possibilités techniques sont quasi illimitées et en évolution permanente, mais que la technique est et sera toujours au service de la communication et non pas l'inverse. 
La stratégie de communication préexiste et prévaut sur un cahier des charges de développement web.

La force des étudiants du DUT Com, dont la formation comporte un volet important lié à la conception d'une stratégie de communication, est donc de connaître la technique, savoir comment s'en servir, et savoir proposer des outils numériques mis au service d'une stratégie.

Les compétences suivantes liées au développement web sont nécessaires :

- La structure du langage html (code source) influe et structure la page de contenu visible par les visiteurs et les performances, l'impact de la communication finale.

- Les principes de structuration touchent au référencement et donc à la visibilité des messages et de la stratégie de communication sur les moteurs de recherche (canal de trafic $n^{\circ} 1$ ).

- Le balisage html lié au référencement naturel, les métadonnées «title » et « description », la structuration du contenu par titre (H1 à H6), les liens internes et externes, l'importance des mots-clefs stratégiques.

- L'aspect technique est aujourd'hui de plus en plus appréhendable fonctionnellement via des interfaces Wysiwyg (What You See Is What You Get) et via des logiciels ou services Saas (Service As A Software) : voir les balises html au sein d'un code source, connaître l'adresse IP d'un serveur, estimer le temps de chargement d'une page, envoyer une newsletter de façon professionnelle, connaître le volume et les sources de visites d'un site, etc.

- Utiliser Google Analytics (analyse de l'audience web), Mailchimp (service Saas d'envoi de newsletter), Dreamweaver pour la conception de pages html, un CMS comme WordPress ou Drupal.

La composante «Graphisme et Contenu » des métiers de la communication web est le segment pour lequel ils ont naturellement le plus d'inclinaison, celui qui leur permet d'exprimer leur créativité. Le graphisme est pour la majorité des étudiants la seule composante "visible » du média web... à tort. Sans minorer son importance, il faut rappeler les étapes d'une communication web vertueuse : être visible / être visité / convaincre / rassurer (cas du e-commerce) / emporter l'adhésion. Le graphisme à toute sa part pour parvenir à ces fins, mais sa part seulement...

La stratégie reste primordiale, sur le plan graphique ou fonctionnel, tout ce qui ne sert pas directement un objectif de communication nuit. Un argumentaire bien écrit et bien structuré est tout autant indispensable, et les qualités rédactionnelles des étudiants sont alors primordiales. Et enfin une stratégie de visibilité et d'acquisition de trafic efficace et qualifiée est toute aussi importante.

Les étudiants du DUT Communication des organisations peuvent ici utiliser l'ensemble des compétences liées à l'infographie et aux techniques rédactionnelles qui est une partie importante de leur formation. Et la primauté de la stratégie ne se 
dément pas, elle est encore, sur cette composante des métiers de la communication web, un point fort des étudiants du DUT Com.

Le Webmarketing est la partie inconnue, dont les étudiants ne soupçonnent ni l'importance, ni la densité, ni la richesse. Les étudiants connaissent tous les encarts publicitaires (images ou vidéo), qui sont à l'identique de la presse papier, affichage, ou TV, l'e-mailing, par expérience personnelle de destinataire, et les médias sociaux qu'ils utilisent au quotidien. Par contre, ils ne les maîtrisent pas, ils ne connaissent pas assez leurs dangers et inconvénients éventuels, ni leur modèle économique, comme par exemple, la monétisation des bases de données utilisateurs par les plateformes sociales.

Les étudiants ne connaissent pas les moteurs de recherche comme encart de visibilité (pourtant $n^{\circ} 1$ ) de référencement naturel ou payant (liens sponsorisés), le « retargeting » ou re-adressage publicitaire qui consiste à re-solliciter l'internaute via un message secondaire après dépôt de cookies au sein de son navigateur. Les notions de « cible qualifiée », de « support qualifié » et de Retour sur investissement (ROI) leur sont inconnues.

Dans le monde idéal de l'étudiant, il suffirait de proposer des communications sur l'ensemble des supports web pour qu'une communication soit réussie et impactante. Certes, mais cela est sans tenir compte des coûts d'achat médias, du temps passé sur de telles pratiques, et sans mettre le doigt sur la notion de ROI et de communication rentable. Les étudiants ont des difficultés à appréhender la communication web comme un système média avec un modèle économique. Ils pensent à tort que le web est gratuit.

La notion de gratuité de consultation des contenus pour les internautes cache sans doute aux étudiants l'existence de logiques économiques d'acteurs. Un support (de préférence, qualifié) met en rapport l'émetteur du message (l'organisation communicante) et la cible finale (consommateur particulier ou b2b). Cette médiation proposée par le support est payante dans les cas de campagnes liens sponsorisés (ex : Google Adwords), d'achat d'espace publicitaire (ex: lefigaro.fr), de produits proposés sur des comparateurs de prix ou places de marché (ex: leguide.com, Cdiscount, Ebay, Amazon), de e-mailing (location de base d'adresses) ou de campagnes sponsorisées sur les médias sociaux.

Pour conclure, la condition d'internaute «surfeur heureux et consommateur de contenus gratuits » de tout étudiant nuit à sa projection dans son futur métier de communicant professionnel sur ce média. Le DUT devra amener les étudiants à une prise de conscience du modèle économique du web.

Les étudiants du DUT Com ont comme points forts leurs compétences en infographie, en techniques rédactionnelles et en création de stratégie de communication. La finalité de ces enseignements devrait tendre à faire des étudiants des «chefs de chantier communication web » susceptibles de comprendre la logique, l'utilité, les conditions et coûts de déploiement de tel ou tel levier web. 


\section{Perspectives}

En conclusion, le travail d'élaboration d'un programme pédagogique est une opération complexe. C'est un chantier jamais fini où savoirs et savoir-faire interagissent pour toujours plus de dialogue entre les communautés universitaires et professionnelles. L'entretien d'explicitation, selon la méthode de P.Vermersch, sera alors très utile pour faire émerger des compétences, valider les savoirs et savoir-être ajoutés dans les modules du nouveau programme sur la base des savoir-faire listés dans le référentiel de compétences.

En 2013, pour la première fois en France, un référentiel des métiers de la communication a été élaboré par les associations et syndicats professionnels du secteur de la communication ${ }^{119}$. Ce travail remarquable, véritable panorama de la profession, va permettre un échange entre professionnels et universitaires sur les compétences identifiées de part et d'autre. En effet, les deux référentiels, qui ont vu le jour en 2013 sans concertation entre eux, mériteraient d'être analysés et harmonisés. L'entretien d'explicitation pourrait être alors utilisé pour permettre aux professionnels d'expliciter leurs compétences et, à partir de leurs savoir-faire, d'identifier les savoirs théoriques réellement utiles à l'exercice de leur profession.

Par la suite, la mise en place du programme de formation, de méthodes de pédagogie active et l'évaluation des formations doit aboutir à un cercle vertueux d'amélioration constante de ces formations. En tant qu'enseignant ou professionnel du secteur de la communication, il est de notre devoir d'exercer une veille active et permanente sur le secteur web pour transmettre et anticiper les évolutions, rapides, et les nouveautés, nombreuses, structurantes pour la discipline. Ceci est aussi le gage de formations professionnalisantes utiles pour l'insertion professionnelle des étudiants.

Ce nouveau PPN, nous l'espérons, permettra de faire reconnaître l'intégration du web et des TIC dans le DUT Communication des organisations. Un effort de communication sur cette évolution doit être mené prochainement par l'ACD Information-Communication. C'est à ce prix que le DUT InformationCommunication renforcera sa place dans le marché européen de la formation, suivant en cela l'harmonisation de l'enseignement supérieur voulue par le processus de Bologne.

J'aimerais citer Jean-Jacques Dijoux, de l'Association de gestion des formations en alternance pour les PME (AGEFA-PME), qui, en guise de conclusion d'une table ronde lors du $4^{\mathrm{e}}$ salon Admission Post-Bac 2014, a soulevé :

l'importance que les formations ne soient pas porteuses du geste professionnel mais bien d'une culture professionnelle, c'est-à-dire qu'elles soient moins de

119 http://metiersdelacommunication.fr/ consulté le 28 septembre 2014 
simples formations utilitaires qu'une capacité pour les jeunes à évoluer et à trouver leur place dans l'entreprise.

Et pour finir, V. Lépine, F. Martin-Juchat et C. Millet-Fourrier (Lépine et al, 2014 : 232) nous offrent le fruit de leur travail en conclusion de l'ouvrage « Acteurs de la communication des entreprises et organisations » :

\begin{abstract}
Si la technicisation des métiers et des activités de communication est incontestable, il n'en est pas moins vrai que la compréhension fine des environnements politiques, économiques, sociaux et culturels dans lesquels se déploient les communications organisationnelles est aussi indispensable. La constante adaptation des formations, le développement des compétences tout au long des parcours professionnels ainsi que la réflexivité des acteurs sur leurs pratiques et le déploiement maîtrisé de régulations éthiques et déontiques, sont autant d'enjeux face auxquels il nous parait indispensable de mobiliser étudiants, enseignants, chercheurs et professionnels.
\end{abstract}

\title{
Bibliographie
}

Claudel, P. (1907). Art poétique, Paris : Mercure de France

Colin, C. et Mouillet, M-C. (2000). Chemin faisant Tome 2, Paris : EAP (Éditions et Applications Psychologiques)

Coutant, A. et Stenger, T. (2011). Community management et community managers : Cheval de Troie marketing pour le web social ?, Actes du 79e congrès international ACFAS «Web social, communautés virtuelles et consommation », 11 mai 2011, Chaire de relations publiques et communication marketing UQAM, Université de Sherbrooke, [En ligne], 140-155, http://www.crpcm.uqam.ca/pages/docs/centres/cmark/ACFAS_428_Actes_Com Mkg_2011.pdf, consulté le 13 septembre 2014

Coyette, C., Lafrance, A. et Lambotte, F. (2013). Les dispositifs de professionnalisation des formations universitaires en communication. Cahiers du RESIPROC, (1), 90-109.

Haulet, D., Lecomte, N., Patesson, R. et Steinberg, P. (2002) Ergonomie et analyse de l'activité, Université Libre de Bruxelles - Centre de Recherches en Ergonomie Appliquée aux Technologies de l'Information et de la Communication, http://www.ulb.ac.be/soco/creatic/ergon1.html, consulté le 12 septembre 2014

Kiyindou, A. (2009). Symposium «Écrire la compétence en information et communication », 8-9 octobre 2009, Roubaix, co-organisé par Les Archives Nationales du Monde du Travail, l'Université Lille 3, la SFSIC et l'École des Hautes Études en Santé Publique, [Retranscription des échanges en ligne], 50- 
178 Le communicateur bousculé par le numérique

52 ,

http://www.sfsic.org/index.php/services300085/bibliotheque/evenements/209-symposium-roubaix2009, consulté le 12 septembre 2014

Lépine, V., Martin-Juchat, F. et Millet-Fourrier, C. (2014). Conclusion générale. Dans V. Lepine et al. (dir.). Acteurs de la communication des entreprises et organisations: Pratiques et perspectives, 229-232, Presses Universitaires de Grenoble

Malric, M. et Vidalenc, I. (2013). L'entretien d'explicitation : Un outil performant pour l'analyse des compétences. Epistémè, Culture, Identity and Digital Writing, F. Liénard (Coord.), (9), 341-362, Center for Applied Cultural Sciences: Université Koréa

Ruedin, Y-M. (2001). ROME, un référentiel des métiers et des emplois. PANORAMA, 3/2001, 16-17, consulté le 6 mai 2013, http://panorama.ch/pdf/2001/Heft_3_2001/heft_3_2001.pdf

Vermersch, P. (2011). L'entretien d'explicitation, Issy-les-Moulineaux : ESF Editeur, collection Pédagogies

Wittorski, R. (2009). Symposium «Écrire la compétence en information et communication ", 8-9 octobre 2009, Roubaix, co-organisé par Les Archives Nationales du Monde du Travail, l'Université Lille 3, la SFSIC et l'École des Hautes Études en Santé Publique, [Retranscription des échanges en ligne], 3-6, http://www.sfsic.org/index.php/services-300085/bibliotheque/evenements/209symposium-roubaix 2009 , consulté le 12 septembre 2014

\section{Webographie}

http://www.iut.fr/ consulté le 28 septembre 2014

http://www.centrale-iut.net/ consulté le 28 septembre 2014

http://www.iutenligne.net/ consulté le 28 septembre 2014

http://www.afci.asso.fr/sites/default/files/pdf/publication/referentiel_afci.pdf consulté le 28 sept. 2014

http://www.enseignementsup-recherche.gouv.fr/cid61532/consultation-sur-lesreferentiels-licence.html consulté le 12 septembre 2014

http://www.pole-emploi.fr/candidat/le-code-rome-et-les-fiches-metiers-

@ (suarticle.jspz?id=15734 consulté le 21 septembre 2014

http://www.iut.fr/publications/le-devenir-des-etudiants.html consulté le 21 sept. 2014 
http://www.enseignementsup-recherche.gouv.fr/cid55933/presentation-autonomiedes-universites.html consulté le 12 sept 2014

http://www.vae.gouv.fr/ consulté le 6 octobre 2014

http://www.anact.fr/web/dossiers/travail-developpement-des-personnes/gpec consulté le 24 septembre 2014

http://metiers.internet.gouv.fr consulté le 28 septembre 2014

http:/cache.media.enseignementsup-

recherche.gouv.fr/file/30/15/0/Infocom_262150.pdf consulté le 28 septembre 2014

http://metiersdelacommunication.fr/ consulté le 28 septembre 2014 